\title{
LMS Adaptive Filtering with Multirate Observations
}

\author{
Anthony H. Hawes and Charles W. Therrien \\ Department of Electrical and Computer Engineering \\ Code EC/Ti \\ Naval Postgraduate School \\ Monterey, CA 93940 \\ Tel: 831.656.3347 FAX: 831.656.2760 \\ e-mail: therrien@nps.navy.mil
}

\begin{abstract}
This paper addresses the problem of adaptive filtering using the LMS method when two or more observation sequences are available with differing sampling rates. In such cases the steady-state behavior of the filters would be periodically time-varying. We develop the form of the algorithm and examine the performance of the algorithm in the context of interference suppression for narrowband and broadband sources of interference.
\end{abstract}

\section{INTRODUCTION}

In related work [1-4] we have investigated the problem of optimal filtering when the signal to be estimated and the observations are not necessarily sampled at the same rate. We have considered both the theoretical formulation of the problem in terms of correlation parameters $[2,4]$ and a least squares formulation of the problem [1] directly in terms of the data. In either case, the optimal linear filter is periodically time varying (LPTV) with period determined by the rates of the observations and the rates at which the desired signal needs to be estimated.

\section{BACKGROUND}

The LMS algorithm plays a significant role in adaptive filtering. The algorithm offers a low cost, elegant solution to adaptive filtering. An arbitrary initial set of filter coefficients is chosen and updated at each time step $n$ and new observation. If the data is wide-sense stationary and the algorithm step size parameter (see below) is chosen appropriately, the filter coefficients approach the optimal coefficients defined by the Wiener-Hopf equations. The nature of this convergence is well-known and discussed in many places (e.g, $[5,6])$. If the data is non-stationary and slowly time--varying, the filter coefficients tend to 'track' the optimal time--varying filter for the data.

Let $d[n]$ represent the signal to be estimated and $\mathbf{x}[n]$ represent a set of observations such that $\hat{d}[n]=\mathbf{w}^{T} \mathbf{x}[n]$. In the basic LMS method the filter coefficient vector $\mathbf{w}$ is updated at each time step $n$ according to the equation

$$
\mathbf{w}[n+1]=\mathbf{w}[n]+\mu e[n] \mathbf{x}[n]
$$

where

$$
e[n]=d[n]-\mathbf{w}^{T}[n] \mathbf{x}[n]
$$

is the error found at time step $n$ and $\mu$ is a parameter called the 'step size.' For suitably chosen values of the step size, the weights converge to a solution close to that of the optimal (Wiener) filter. For practical purposes, the bounds on the step size can be approximated as [5]

$$
0<\mu<\left[2 /\left(P \cdot R_{x}[0]\right)\right]
$$

where $P$ is the order of the filter and $R_{x}[0]$ is the value of the autocorrelation sequence of the input signal at lag zero (the signal power).

\section{THE MULTIRATE LMS ALGORITHM}

The multirate LMS estimation algorithm extends the LMS algorithm to the case where multiple inputs at different sampling rates are available. Accordingly, for the multirate case, the equations are slightly more complicated. In this discussion it is assumed, that two inputs are available (see Fig. 1) and these are to be used jointly to estimate the 'desired' signal $d[n]$.

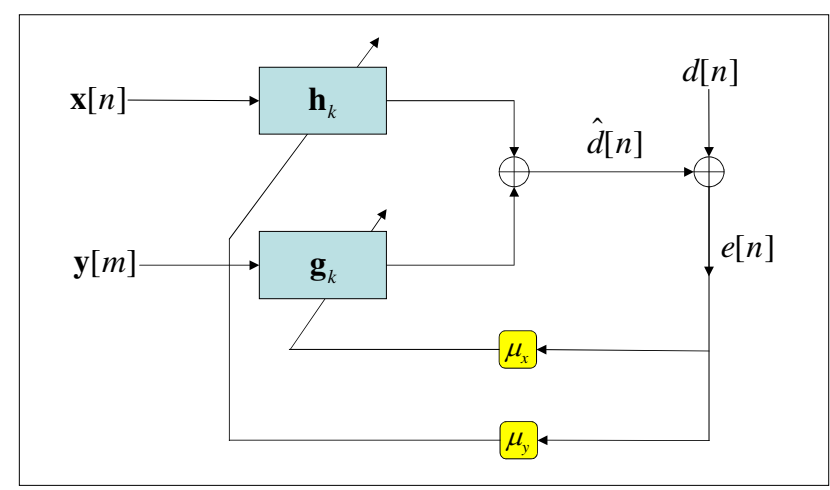

Fig. 1. Simplified Diagram of Multirate LMS Algorithm

The input $\mathbf{x}$ is sampled at the full rate, i.e., the rate of the output estimate. The input $\mathbf{y}$ is sampled at a rate of $1 / K$ times the full rate. The terms $\mathbf{h}_{k}$ and $\mathbf{g}_{k}$ represent vectors of filter coefficients associated with the high-rate and low- 
rate filters, respectively. Let $m$ represent the time index of the low rate signal. Then at any time $n$, one can write $n=K m+k$ for $k=0,1, \ldots, K-1$. Here $m=\lfloor n\rfloor$ is the smallest integers less than or equal to $n$.

Let us define a vector of high-rate data points as

$$
\mathbf{x}[n]=\left[x[n] \quad x[n-1] \quad \cdots \quad x[n-(P-1)]^{T}\right.
$$

and a corresponding vector of low-rate data points as

$$
\mathbf{y}[m]=\left[\begin{array}{llll}
y[m] & y[m-1] & \cdots & y[m-(Q-1)
\end{array}\right]^{T}
$$

where $P$ and $Q$ are the orders of the two FIR filters represented by $\mathbf{h}_{k}$ and $\mathbf{g}_{k}$ in Fig. 1 . The estimate is then of the form

$$
\hat{d}[n]=\mathbf{h}_{k}^{T} \mathbf{x}[n]+\mathbf{g}_{k}^{T} \mathbf{y}[m] ; \quad n=K m+k .
$$

In the absence of adaptation the filter coefficients would be periodic $[1,2]$, i.e., at any times $n_{1}$ and $n_{2}$ such that $n_{1}(\bmod K) \equiv n_{2}(\bmod K) \equiv k$ the coefficient vectors $\mathbf{h}_{k}$ and $\mathbf{g}_{k}$ are independent of the time index $n$ or $m$. For the multirate LMS algorithm these coefficient vectors are updated in time. The coefficient vectors at any time $n$ will be denoted by $\mathbf{h}_{k}[m]$ and $\mathbf{g}_{k}[m]$ (where $n$ has been represented as $n=K m+k$ ). The estimate is thus given by

$$
\hat{d}[n]=\mathbf{h}_{k}^{T}[m] \mathbf{x}[n]+\mathbf{g}_{k}^{T}[m] \mathbf{y}[m] ; n=K m+k .
$$

The error is then given by

$$
e[n]=d[n]-\hat{d}[n] .
$$

The update steps in the multirate LMS algorithm are defined by

$$
\mathbf{h}_{k}[m+1]=\mathbf{h}_{k}[m]+\mu_{x} e[n] \mathbf{x}[n]
$$

and

\begin{tabular}{|c|}
\hline CHOOSE $\quad n_{0}, m_{0}$ \\
\hline$i=0$ \\
\hline $\mathbf{y}[i]=\left[\begin{array}{lll}y\left[m_{0}+i\right] & \cdots & y\left[m_{0}+i-Q+1\right]\end{array}\right]$ \\
\hline$k=0$ \\
\hline$d_{k}[i]=d\left[n_{0}+i K+k\right]$ \\
\hline $\mathbf{x}_{k}[i]=\left[x\left[n_{0}+i K+k\right] \quad \cdots \quad x\left[n_{0}+i K+k-P+1\right]\right]$ \\
\hline$\hat{d}_{k}[i]=\mathbf{h}_{k}^{T}[i] \mathbf{x}_{k}[i]+\mathbf{g}_{k}^{T}[i] \mathbf{y}[i]$ \\
\hline$e_{k}[i]=d_{k}[i]-\hat{d}_{k}[i]$ \\
\hline $\mathbf{h}_{k}[i+1]=\mathbf{h}_{k}[i]+\mu_{x} e_{k}[i] \mathbf{x}_{k}[i]$ \\
\hline $\mathbf{g}_{k}[i+1]=\mathbf{g}_{k}[i]+\mu_{y} e_{k}[i] \mathbf{y}[i]$ \\
\hline$k=k+1$ \\
\hline UNTIL $k>(K-1)$ \\
\hline UNTIL $\quad i>M$ \\
\hline
\end{tabular}

$$
\mathbf{g}_{k}[m+1]=\mathbf{g}_{k}[m]+\mu_{y} e[n] \mathbf{y}[m]
$$

where $\mu_{x}$ and $\mu_{y}$ are step size parameters.

The complete algorithm is listed in Fig. 2 and depicted graphically in Fig. 3.
Fig.2. Flow Diagram for Multirate LMS Algorithm

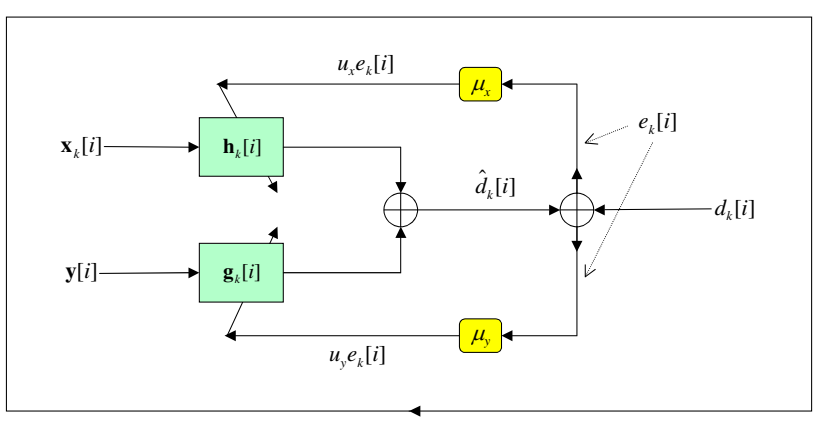

Fig.3. Diagram of Multirate LMS Algorithm

Since the time-varying data filters have period $K$, there are $P K$ weights needed for the high-rate filter and $Q K$ weights needed for the low-rate filter. For the high-rate case, if we define a $P$ by $K$ matrix containing all highrate coefficients

$$
\mathbf{H}[i]=\left[\begin{array}{cccc}
\vdots & \vdots & & \vdots \\
\mathbf{h}_{0}[i] & \mathbf{h}_{1}[i] & \cdots & \mathbf{h}_{K-1}[i] \\
\vdots & \vdots & & \vdots
\end{array}\right]
$$

whose columns represent the filter at each step $k=0,1, \ldots, K-1$ in its period, then we see that only one column of $\mathbf{H}[i]$ is updated for each point of the output sequence that is estimated (see Fig. 2). Beginning the 
estimation at corresponding points $n_{0}$ and $m_{0}$ of the observation sequences, the filter $\mathbf{h}_{0}[0]$ is found. Then the input matrix $\mathbf{x}_{k}[i]$ is updated and used to find $\mathbf{h}_{1}[0]$. The process continues until all $K$ columns of $\mathbf{H}[i]$ have been updated, at which time the cycle repeats. The coefficients for the $k^{\text {th }}$ step in the cycle of the time-varying filter $\mathbf{h}_{k}$ are therefore updated at every $K^{\text {th }}$ point of the original sequence.

A similar discussion applies to the low-rate coefficients. The complete set of coefficients are contained in a $Q$ by $K$ matrix

$$
\mathbf{G}[i]=\left[\begin{array}{cccc}
\vdots & \vdots & & \vdots \\
\mathbf{g}_{0}[i] & \mathbf{g}_{1}[i] & \cdots & \mathbf{g}_{K-1}[i] \\
\vdots & \vdots & & \vdots
\end{array}\right] .
$$

A particular column $\mathbf{g}_{k}$ is then updated at every $K^{\text {th }}$ point of the original data sequence.

\section{SIMULATION PRELIMINARIES}

The multirate LMS filter was tested in the context of adaptive interference cancellation (also known as adaptive noise cancellation or ANC). Results are given for a speech signal with both narrowband and broadband interference. For the basic, single-rate LMS ANC, the primary input contains the signal plus interference. The so-called 'reference' input contains a correlated version of the interference that is used by the adaptive filter to cancel the interference in the primary input.

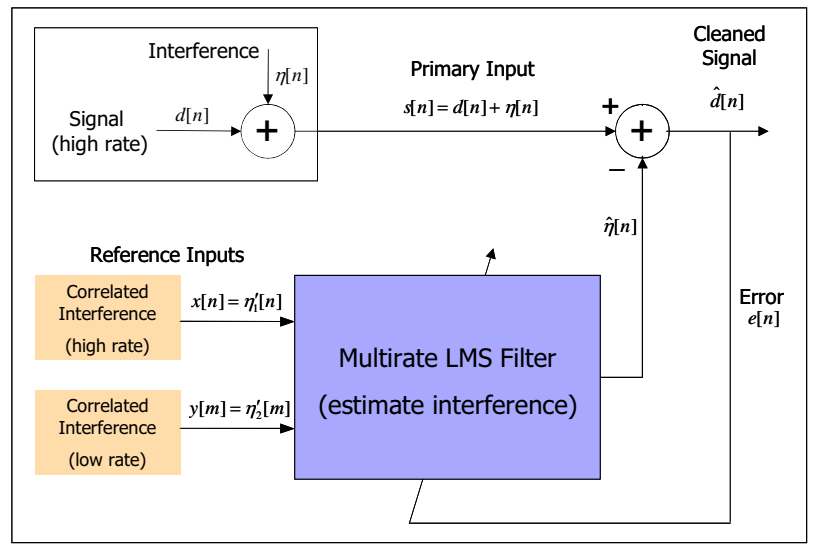

Fig. 4. Adaptive Interference Cancellation with Two Reference Inputs

The ANC extended to the case of multiple reference inputs is shown in Fig. 4. The inputs to the filter now contain multiple versions of the interference, sampled at different rates. The goal of the LMS filter is to estimate the interference present in the reference input (at the full rate) from two noisy independent observations of the interference.

A case of some interest is where the only reference signal is the lower rate signal $y[m]$. In other words, the reference signal is available at only a lower rate compared to the primary input $s[n]$ and the cleaned signal $\hat{d}[n]$. In this case it may be able to perform the ANC with fewer samples (in time) of the correlated interference.

\section{SIMULATION RESULTS}

Results for both narrowband and broadband interference are presented in this section. Further details of the simulations can be found in [7].

\section{A. Narrowband Interference}

The test signal for this experiment was a recording of the spoken word 'hello' with a prolonged 'o.' This sample of speech is two seconds long and sampled at $22.05 \mathrm{kHz}$; the waveform is shown in Fig. 4(a). The interference consisted of tonal 'noise' added to the recorded speech, i.e., two sinusoidal tones were added to the signal. A tone of 4.41 $\mathrm{kHz}$ was added at the beginning of the speech segment. At the midpoint of the speech segment, this first tone was ended and a second tone of $2.205 \mathrm{kHz}$ was added for the remainder of the speech signal. The original speech signal and the corrupted version are shown in Fig. 5.
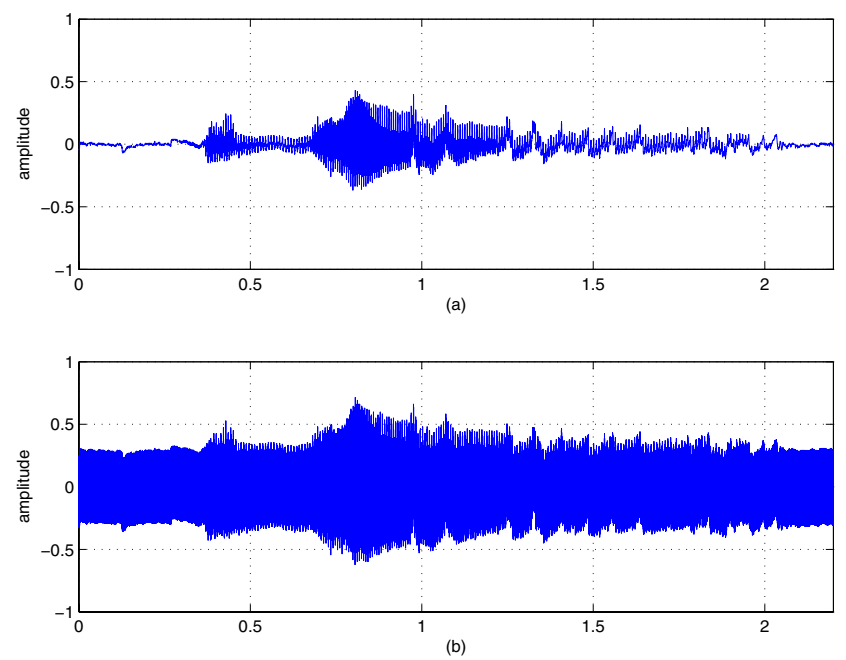

Fig. 5. Plot of voice signal of the spoken word 'hello' used in narrowband interference simulation. (a) Original speech signal. (b) Speech signal including tones. 
The reference input was generated by sending the interference signal through a third-order FIR filter, with coefficients chosen randomly, and downsampling the result.

Since the interference is sinusoidal, the order of both filters was taken to be $P=Q=2$. The number of samples required for the filter to mute the initial sinusoid is used as a measure of speed of convergence below.

\section{(1) Using Both Observation Signals}

The corrupted signal was filtered using the parameters described above; a sampling rate ratio of $K=4$ was used. The resulting MSE was $-14.15 \mathrm{~dB}$. It took approximately 4,575 samples or $210 \mathrm{~ms}$ to mute the initial sinusoid in this case. While this is slow for channel equalization in a communications system, it may suffice for many audio applications. The filtered signal is shown in Fig. 6(b) and can be compared to the original signal in Fig. 6(a). The spikes in the sequence at samples zero and 25,000 are the points at which the sinusoids were added.

\section{(2) Using Low-rate Observations Alone}

In this case, the corrupted signal is filtered using the low-rate observation sequence as the only reference input. The low-rate observation sequence has a sampling rate of $5.51 \mathrm{kHz}$. The multirate LMS filter estimates the interference at the full rate of $22.05 \mathrm{kHz}$, using these samples. All other simulation parameters are the same as in the previous case. The filtered signal is shown in Fig. 6(c). For this case, the filter took approximately 6550 samples or $300 \mathrm{~ms}$ to mute the initial sinusoid. The resulting MSE was $-12.91 \mathrm{~dB}$.

This experiment was also performed using a longer segment of speech taken from the cockpit of a military jet; similar results were obtained.
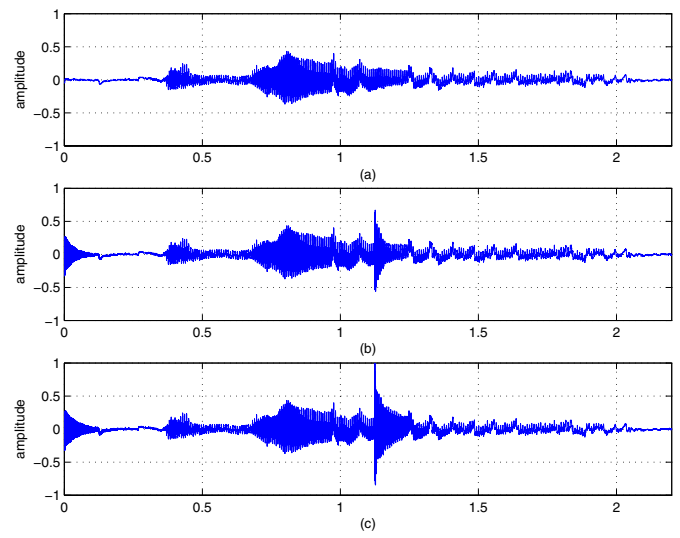

Fig. 6. Signals after filtering. (a) Original speech. (b) Filtered using both observations (c) Filtered using low-rate observations

\section{B. Broadband Interference}

An adaptive interference scenario was also used for this scenario. The interference was a spoken phrase of speech, which is a broadband signal. The interference was removed using the low-rate observations alone. The desired signal for this experiment was a segment of radio traffic from an air traffic control center. The reference input was generated by sending the interference signal through an FIR filter with randomly-selected coefficients and then downsampling the resulting sequence by a factor of $K$. The parameters for this simulation were: the order of the low-rate filter $Q=40$, the low-rate SNR was $S N R_{\text {low }}=-0.26 \mathrm{~dB}$, the sampling rate ratio was $K=4$. The SNR of the filtered sequence was $9.65 \mathrm{~dB}$, which is an improvement of $9.91 \mathrm{~dB}$.

\section{CONCLUSIONS}

The LMS algorithm of adaptive filtering is extended to the case of multiple observation sequences where one or more of the input rates is different from the output rate. The method has been tested in an interference cancellation (ANC) application for speech with narrowband and broadband sources of interference. A particular case of interest is where there is only a single reference input for the interference sampled at a lower rate compared to the original and interference. Experimental results show that satisfactory performance can be achieved with a rate reduction (decimation factor) of four.

\section{REFERENCES}

[1] Charles W. Therrien and Anthony H. Hawes, "Least squares optimal filtering with multirate observations," Proc. $36^{\text {th }}$ Asilomar Conf. On Signals, Systems, and Computers, November 2002, pp. 1782-1786, Pacific Grove, CA.

[2] Charles W. Therrien and Ryan J. Kuchler, "Optimal filtering with multirate observations," Proc. $37^{\text {th }}$ Asilomar Conf. On Signals, Systems, and Computers, November 2003, Pacific Grove, CA.

[3] C. W. Therrien, "Issues in multirate statistical signal processing," Proc. $35^{\text {th }}$ Asilomar Conf. On Signals, Systems, and Computers, November 2001, pp. 573-576, Pacific Grove, CA.

[4] R. Cristi, D. Koupatsiaris, and C. W. Therrien, "Multirate filtering and estimation: the multirate Wiener filter," Proc. $34^{\text {th }}$ Asilomar Conf. On Signals, Systems, and Computers, October 2000, pp. 450454, Pacific Grove, CA

[5] Simon Haykin, Adaptive Filter Theory, Prentice Hall, Inc., 4th Edition, Upper Saddle River, New Jersey, 2002.

[6] O. Macchi, Adaptive Processing: the Least Mean Squares Approach, John Wiley and Sons, 1995.

[7] Anthony H. Hawes, "Least Squares and Adaptive Multirate Filtering," Master's thesis, Naval Postgraduate School, Monterey, California, 2003. 